\title{
Origin of "Very-Low-Frequency Emissions"
}

\author{
R. M. Gallet and R. A. Helliweli
}

(January 16, 1959)

\begin{abstract}
Selective traveling-wave amplification in the outer ionosphere is postulated to explain very-low-frequency emissions, a class of very low-frequency ( 1 to 30 kilocycles per second) natural noise. By analogy with the mechanism of traveling wave tubes, low-level ambient noise in the outer ionosphere is amplified in streams of incoming ionized solar particles at frequencies for which the stream and wave velocities are equal. Required velocities are in the range 0.01 to $0.1 c$ (where $c$ is the velocity of light). Streams with densities of the order of one electron per cubic centimeter would provide sufficient energy. Phenomena which can be explained qualitatively by the theory are the hiss, quasi-constant tones, dawn chorus and related transients, and very long trains of whistler echoes. A quantitative example shows how the theory can reproduce the general form of certain characteristic discrete spectra "hooks" of emissions, and how this leads to definite values of particle velocity and a law for the distribution of electron density in the outer ionosphere.
\end{abstract}

\section{Introduction}

When a high-gain audio amplifier is connected to a loop or long-wire antenna, unusual sounds of natural origin are often heard. The more impulsive sounds called "atmospherics" are produced by lightning and reach the receiver by traveling between the ground and the lower edge of the ionosphere. They are described by various terms such as "clicks," "tweeks," and "crashes."

Some of the energy from a lightning discharge also enters the ionosphere and is guided by the lines of force of the earth's magnetic field. As the energy propagates from one hemisphere to the other, it is dispersed and arrives at the receiver as a whistling tone, usually of descending frequency. Such signals are called "whistlers," and their theory has been developed in some detail (Storey [1] , Helliwell et al. [2], Ellis [3], and Maeda [4]).

There is yet another class of audiofrequency signals which we will call "very-low-frequency emissions". These appear to be related to magnetic disturbance and occur most frequently near the auroral zones. (Storey [1], Pope [5], Allcock [6], Dinger [7], and Watts [8].) One of the most common is the "niss", a band of noise several kilocycles wide which is usually heard below $10 \mathrm{kc}$; it is occasionally accompained by one or more discrete tones in the same range of frequencies. Another is the so-called "dawn chorus", a combination of short (0.1-0.2 sec) rising whistles and warbling tones. Various isolated events, including rising and falling tones and combinations thereof can be observed. Very-lowfrequency emissions are frequently associated with strong whistlers. Sometimes a whistler appears to "trigger" short periods of dawn chorus or hiss. Whistlers are sometimes preceded or followed by one or more rising tones.

It is the purpose of this paper to outline a theory

1 The basic ideas described in this paper we"e presented to the Symposium on the Propagation of VLF Radio Waves, January 23-25, 1957, Boulder, Colo. 2 Dr. Helliwell is affiliated with the Radio Propagation Laboratory, Stanford University, California.

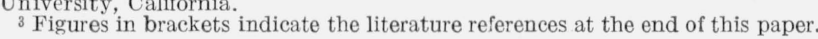

of the origin of vlf emissions, based on selective traveling-wave amplification taking place in the outer ionosphere. The input signal is assumed to be provided by thermal radiation, whistler energy, or possibly Cerenkov radiation (Ellis [9]). It is assumed that the energy for amplification of the electromagnetic wave is provided by the streams of ionized solar particles (both positive and negative) which are thought to cause the auroras. These streams are assumed to penetrate the ambient ionization of the outer ionosphere and to be guided along the lines of force of the earth's magnetic field. The mechanism of amplification is essentially similar to that which takes place in an ordinary traveling wave tube (Pierce [10]), except that the "slow-wave" circuit (provided by the helix in the tube) is the ambient ionization of the outer ionosphere in the presence of the earth's magnetic field. Such a medium is dispersive, i.e., the wave velocity is a function of frequency, and at the very low frequencies considered here the velocity may be reduced as much as two orders of magnitude below that in free space. Furthermore, as shown by Storey [1] in connection with the theory of whistlers the energy of a vlf wave tends to be guided along the earth's magnetic field lines rather closely, and thus may follow the same path as the postulated particle stream. The wave normal, on the other hand, may make any angle with the field; and since the wave polarization is approximately circular, there will normally be a substantial component of the electric field of the wave which is parallel to the magnetic field. This component will act on the moving particle stream to produce the required sinusoidal modulation of charge density. For given values of the ambient plasma and gyrofrequencies, the stream velocity will approximate the phase velocity at two particular frequencies, and traveling wave amplification can take place. As the parameters of the medium change with position along the stream, the frequencies of amplification will change. This paper develops the conditions for interaction assuming the validity of the analogy with the traveling wave tube mechanism. 


\section{Interaction Conditions}

By analogy with the small-signal theory of traveling wave tubes (Pierce [10]), the necessary condition for coupling between the electromagnetic wave and the stream of charge is

$$
v_{p}=v_{s}
$$

where $v_{p}=$ phase velocity of wave, $v_{s}=$ velocity of stream.

We must now deduce the conditions under which (1) will be satisfied. The phase velocity is $c n$, where $c$ is the velocity of light and $n$ is the refractive index in the medium. Since we need a phase velocity less than the velocity of light, we shall be interested in the "whistler-mode" of propagation (Storey [1]). For simplicity we shall consider the case in which the wave normal direction is approximately the same as that of the earth's magnetic field. The refractive index for the propagating mode is then given by

$$
n^{2}=1+f_{p}^{2} /\left\{f\left(f_{H}-f\right)\right\},
$$

and the phase velocity is then simply

$$
v_{p}=c\left[\frac{f\left(f_{H}-f\right)}{f\left(f_{H}-f\right)+f_{p}^{2}}\right]^{1 / 2},
$$

where $f=$ wave frequency; $f_{H}=$ gyrofrequency; $f_{p}=$ $(81 N)^{1 / 2}=$ plasma frequency; with $N=$ number of electrons per cubic centimeter when $f_{p}$ is in kilocycles per second.

It is assumed that $f_{p}>f, f_{H}>f$. From (1) and (2), the frequencies of amplification are given by

$$
f=\frac{f_{H}}{2}\left\{1 \pm\left[1-\frac{\left(\frac{2 v_{s}}{c} \frac{f_{p}}{f_{H}}\right)^{2}}{1-\left(v_{s} / c\right)^{2}}\right]^{1 / 2}\right\} .
$$

If we restrict the discussion to cases in which the stream velocity is less than about $0.1 c$, then $\left(v_{s} / c\right)^{2} \ll 1$, and (3) can be written

$$
f=\frac{f_{H}}{2}\left\{1 \pm\left[1-\left(2 P / f_{H}\right)^{2}\right]^{1 / 2}\right\},
$$

where $P=f_{p}\left(v_{s} / c\right)$ and is conveniently measured in kilocycles per second.

For interaction to occur, the solution to (4) must be real, and hence the condition for interaction is

$$
f_{H} \geqq 2 P \text {. }
$$

The maximum value of the wave frequency $f$ is $f_{H}$, since the maximum real value of the radical in (4) is unity. The conditions for interaction are shown in figure 1, which is a plot of wave frequency $f$ as a function of gyrofrequency $f_{H}$ for different values of the parameter $P$. All curves are asymptotic to the line $f=f_{H}$ (ignoring the dashed curve for the moment).
As an example of the application of these curves, consider a stream of velocity $3,000 \mathrm{kmps}$ which enters the outer ionosphere along an auroral line of force (near geomagnetic latitude $67^{\circ}$ ). The ambient plasma frequency is assumed to be constant and equal to $200 \mathrm{kc}$, and thus the parameter $P=3 \mathrm{kc}$. When the stream reaches the point where $f_{H}=2 P$, interaction can begin at the wave frequency $f_{H} / 2$, or 3 kc. Beyond this point two frequencies can be amplified, and as $f_{H}$ increases the lower one approaches zero and the upper one approaches $f_{H}$, following the curve $P=3$ in figure 1 . The locations of these frequencies along the dipole line of force are plotted in figure 2 .

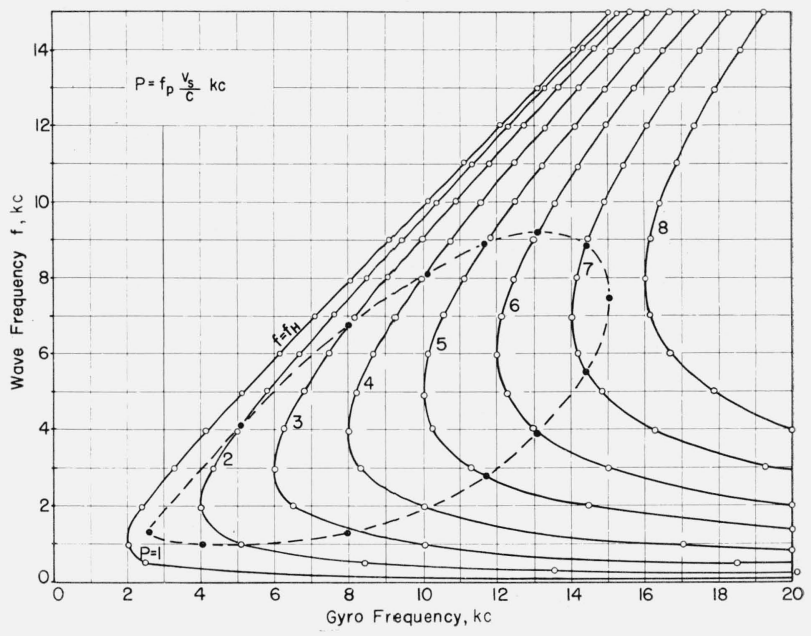

Figure 1. Variation of excited wave frequency with gyrofrequency.

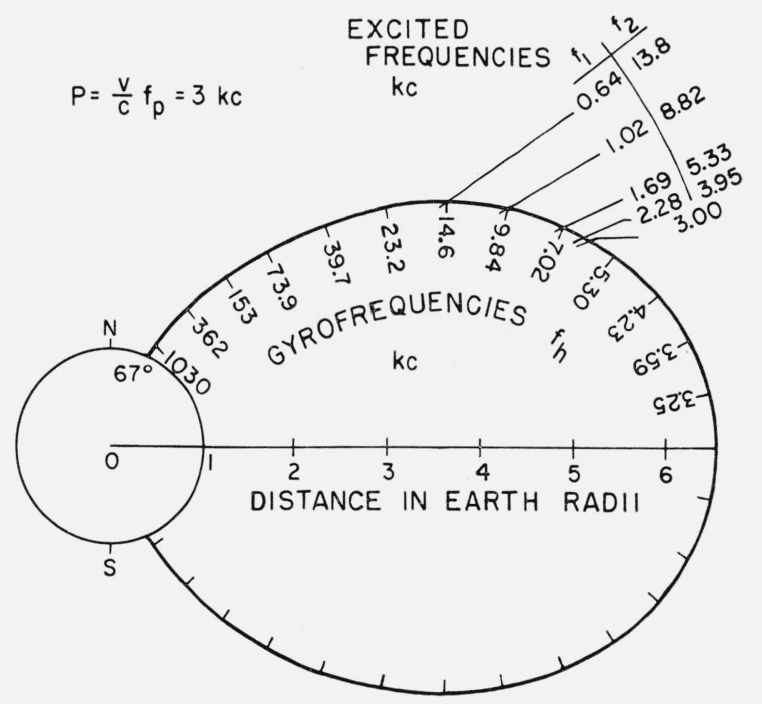

Figure 2. Illustration of the theory of hiss, for the auroral line of force $\lambda=67^{\circ}$. 


\section{Stream Velocities}

The stream velocity required for interaction is found from (4) and is given by

$$
v_{s}=\frac{c}{f_{p}} \sqrt{f\left(f_{H}-f\right)} .
$$

Values for the stream velocity for appropriate values of the medium parameters and the wave frequency are given in table 1 . It is seen that the required stream velocities could vary roughly between 0.01 and 0.1 of the velocity of light. These velocities seem reasonable, since the lower value, $0.01 c$, is comparable to the lower limit found by Meinel [11] in spectroscopic studies of hydrogen in the aurora. On the other hand, it appears necessary that electrons which penetrate to the $E$ region must have velocities of approximately $0.3 c$ (Vegard [12]). Thus the velocities required for the traveling wave mechanism appear to lie in the same range as those ascribed to auroral particles.

\section{Stream Energy Requirements}

If traveling wave amplification is to be accepted as a possible mechanism, it is necessary that the power density of the incoming stream exceed that of the observed signals. A measurement of the strength of relatively strong hiss was made at Stanford, California (Stanford University [13]) on March 3, 1956, during a period of magnetic disturbance. The power density was of the order of $10^{-10} \mathrm{w} / \mathrm{m}^{2}$. for a signal having a bandwidth of about $1 \mathrm{kc}$. Observations of whistlers indicate that the total attenuation from the source to the observer is not likely to exceed $20 \mathrm{db}$. Thus the power density of the signal as it leaves the amplifiying region is of the order of $10^{-8} \mathrm{w} / \mathrm{m}^{2}$.

Let us now calculate the power density of the incoming stream. Since the particle densities and velocities of streams from the sun are not known, we must make a conservative estimate. From the work of Martyn [14] the stream is estimated to have a density of at least 1 electron $/ \mathrm{cm}^{3}$ and a velocity of $3,000 \mathrm{~km} / \mathrm{s}$. The power density in such a stream is $\left(\frac{1}{2} m v^{2}\right)(N v)$, where $m$ is the particle mass, $v$ the velocity, and $N$ the number of particles per unit volume. For electrons, $m=9.11 \times 10^{-31} \mathrm{~kg}$, and the power density of the stream is thus about
$10^{-5} \mathrm{w} / \mathrm{m}^{2}$. The required efficiency of conversion from kinetic energy to electromagnetic energy is thus 0.1 percent, a low value compared with traveling wave tube efficiencies, which are of order 10 percent.

\section{Comparison of Theory With Observations}

Using the traveling wave hypothesis the hiss is explained in terms of a continuous stream of particles along which amplification occurs only for a restricted band of frequencies. The amount of gain at any one frequency will depend upon the interaction distance available, which in turn will depend on the way in which the plasma frequency varies with distance along the path. In a like manner the quasiconstant tones sometimes observed on different frequencies can be explained by assuming an ionization model in which the plasma frequency increases with gyrofrequency in such a way that $f=$ constant over an appreciable length of path. Several different tones observed simultaneously could be explained by different streams flowing along lines of force of sightly different geomagnetic latitude, or possibly by streams of different velocities.

Transient phenomena, of which the dawn chorus is an example, are less easily explained. The traveling wave hypothesis can be adapted to the generation of transient signals by assuming that the incoming particles arrive in bunches. As the bunches travel along the lines of force, amplification takes place in the region of the bunch. However, the characteristic frequencies are amplified for a length of time which depends upon the length and velocity of the bunch. As the bunch moves, the frequency which is generated changes in accordance with changes in the parameters of the medium. The mechanism of formation of such bunches is not known; however, the visual phenomena observed during aurora suggest that solar streams may be bunched.

The interpretation of observed transient signals in terms of bunches of particles is complicated by the fact that the frequency-time relation of the received signal will depend on the integrated group delay from the point of origin to the receiver. The group delay, which is a function of frequency, clearly will affect the frequency-time dependence of the received signal. However, it cannot by itself explain the appearance of a given frequency at two different times, a phenomenon seen frequently in the dawn chorus. Such cases might better be explained in terms of variations in the ambient ioniza-

TABLE 1. Calculated stream velocities for different parameters of amplifying region

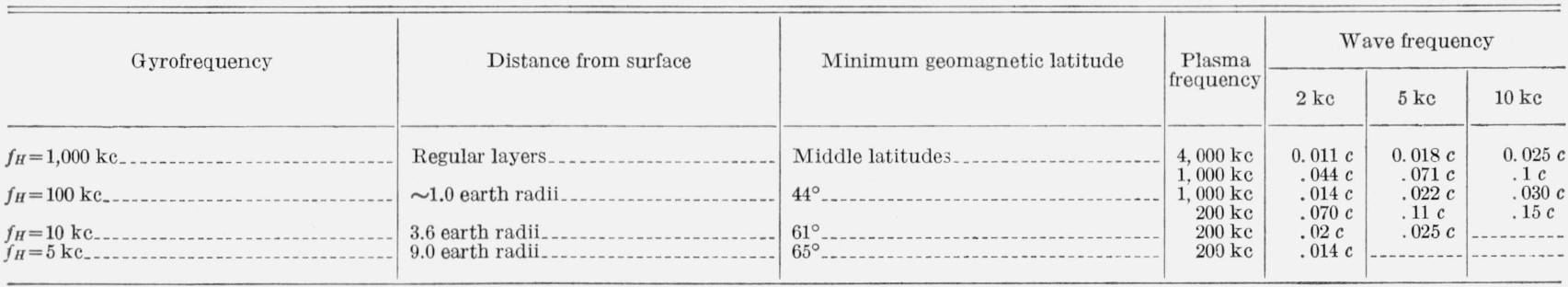


tion density in the interaction region. For example, consider the hypothetical model, $f_{p}=200 \mathrm{kc}+f_{H}{ }^{2}$ ' (1 kc), where $f_{H}$ is measured in kilocycles per second. A short bunch of charge (say, with dimensions of about $100 \mathrm{~km}$ ) traveling through such a region would excite the frequencies given by the dashed curve of figure 1. The energy would be limited to short groups produced at different times. The curve of excited frequency versus gyrofrequency transforms to a somewhat similar shape in the frequency-time plane. Some distortion of this curve occurs at the receiver because group delay is a function of both frequency and location of source. No completely closed trace of this type has yet been observed, but many of the recorded signals first fall and then rise with time, corresponding to the lower portion of the dashed curve. Thus there is at least a qualitative resemblance between the theory and the experimental data.

The steady hiss and the sharply defined transients represent extremes among vlf emission phenomena. In between lie many forms which show varying degrees of irregularity. Their character often changes markedly in the course of a few hours. It is suggested that the observed variety of form is simply the result of variations in the degree of bunching of the charge in the streams.

The traveling wave hypothesis offers a convenient explanation of very long trains of echoes of a whistler. The echo intensity decreases very slowly with order, and may even increase. It is difficult to explain this behavior in terms of the passive properties of the medium. Discrete echoes in a single train have been detected for as long as $4 \mathrm{~min}$. These rather rare events are usually accompanied by magnetic disturbance and the dawn chorus or hiss. The whistler components which are most prolonged often lie in the 3- to 4-kc range where the hiss is known to predominate. On the basis of these facts it is suggested that the low apparent attenuation in very long whistler trains can be attributed to traveling wave amplification somewhere along the path. The whistler energy propagates along the same path followed by the incoming solar stream and is amplified in the regions where the whistler phase velocity approximates the stream velocity.

One of the most puzzling phenomena is the interaction between loud whistlers and vlf emissions. If during a period of dawn chorus and whistler activity, an ordinary loud whistler should occur, it is frequently accompanied by one or more rising whistles (Storey [1]). In attempting to explain this connection in terms of the traveling wave concept, we suggest that the loud whistler may be capable of accelerating an incoming stream of particles. For energy to be transferred from the whistler to the stream, the wave must accelerate the particles in its electrical field. Equation (2) shows that in a region where $f_{p}$ does not increase too rapidly with $f_{H}$, the phase velocity of a fixed frequency wave will increase with $f_{H}$. For our purposes the rate of change of whistler frequency with time is sufficiently small that we can assume the accelerating signal to be of constant frequency. Thus as the whistler travels down the field line, particles of a given velocity are literally picked up by the wave at every point where a wave component of the same velocity exists. As the wave continues to propagate, its phase velocity increases and the particles riding on the front of the wave are accelerated, as in a linear accelerator. With the additional kinetic energy which the stream acquires from the signal it would be more efficient as a traveling wave amplifier and might conceivably produce audible vlf emissions.

\section{Conclusions}

The rather close similarity between conditions in the outer ionosphere and those in a traveling wave tube lead to the hypothesis that traveling wave amplification might take place at very low audiofrequencies. Even though the traveling wave hypothesis may not account successfully for all of the observed phenomena, it is probably an important factor to be considered in whistler propagation as well as in the generation of vlf emissions. Confirmation of the hypothesis must await a more detailed study of the traveling wave-gain problem and somewhat more detailed information regarding the distribution of ionization in the outer ionosphere. Such data presumably will come from whistler analysis. This theory, if correct, should provide a useful new tool for a study of the densities, velocities, and locations of streams of charged particles in the outer ionosphere.

\section{Appendix: A Quantitative Comparison of the Theory With Observation}

\subsection{Example Chosen for Comparison With the Theory}

The theory presented above has been developed in extensive numerical applications by one of us (R. M. G.) to see if the theory is able to account in detail for spectra of discrete vlf emissions, using a minimum of reasonable assumptions.

There is considerable information on the distribution of electron density up to the height of maximum density in the ionosphere, but very little is known about densities above this height. In particular, the law governing the distribution of electron density with height is not known for this region. In these investigations several models of the distribution above the main body of the ionosphere were considered. They were: Constant electron density; a power law $r^{-n}$ where $r$ is the distance from the center of the earth and $n$ was taken from 1 through 6 ; and an exponential distribution $e^{-k T}$ where $k$ is a constant. The power law was found to give the best representation of certain distinguishing features of the vlf emissions and also to be consistent with information obtained from parallel studies of whistlers 
(dispersion constants and analysis of nose whistlers). The same criteria seem to require the exponent $n \simeq 3$. Furthermore, a power law distribution with $n \simeq 3$ appears to be justified theoretically in other research in progress on the gravitational equilibrium of extended atmospheres, at large distances from the earth's surface. It is perhaps significant for the physics of the exosphere that $n \simeq 3$ means that the electron density is nearly proportional to the strength of the earth's magnetic field.

An example of a computed spectrum (frequency versus time at the earth's surface) of waves emitted by a discrete cloud of ionized particles moving with constant speed parallel to the lines of force of the earth's magnetic field is shown in the upper part of figure 3. This example was chosen to approximately match the observed spectrum shown in the lower part of the figure. The latter is a typical member of the "Hook" class of vlf emissions. This class is one of the best defined, and the reproducibility of the emissions is remarkably good in general shape. However, no two hooks, even though separated by only a few seconds in time, are exactly the same. Therefore, the exact matching of any individual tone is of no particular importance. In the example shown here, no great accuracy in matching was attempted; the same computations made with a slightly lower speed for the cloud of particles (say $9,000 \mathrm{~km} / \mathrm{sec}$ ) would have resulted in a better match.

It is more significant to realize that with reasonable numerical values for the parameters, the theory leads very naturally to calculated spectra very similar to those observed in regard to values of frequency, duration, and general shape.
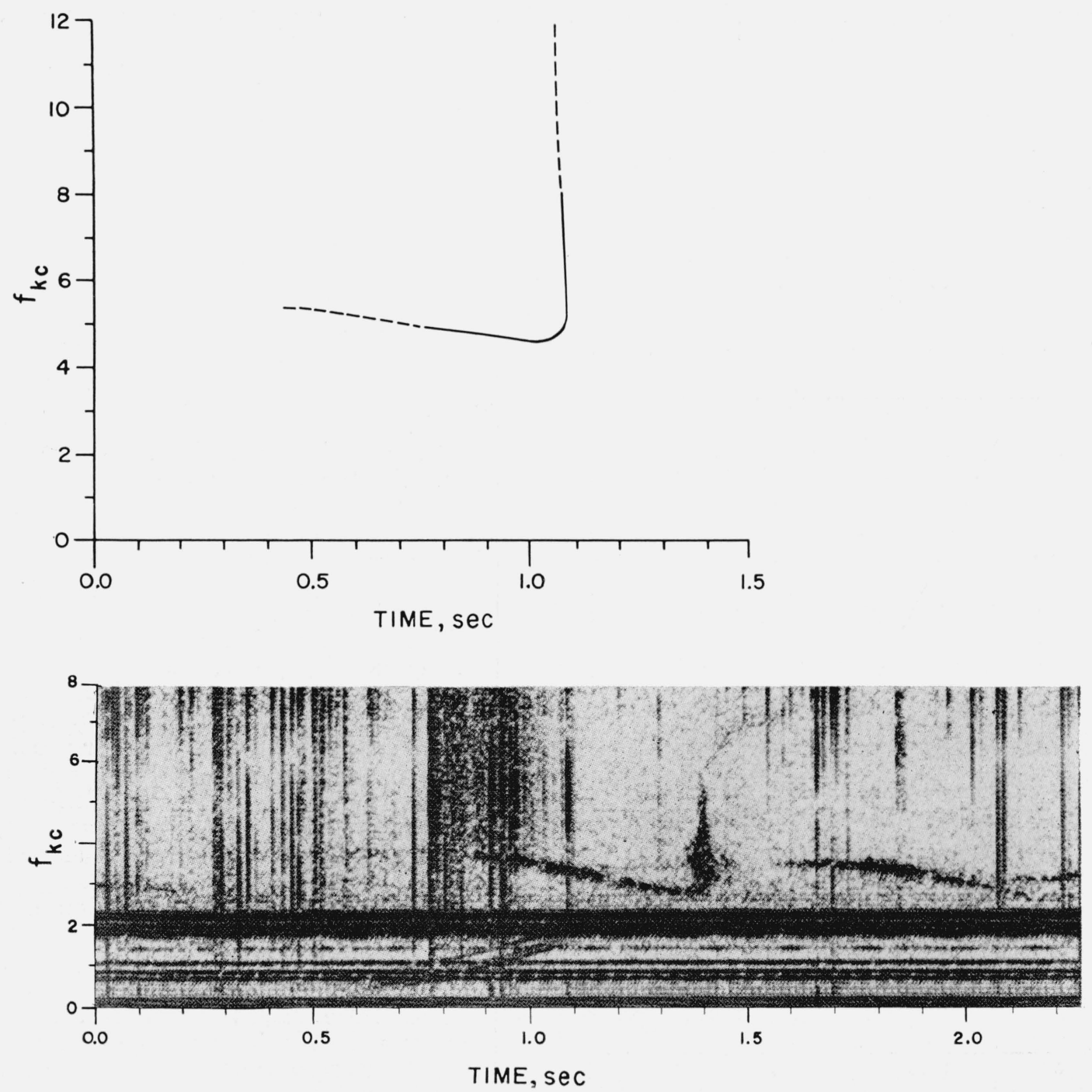

Figure 3. Comparison of theory and observation (see appendix for details). 


\subsection{Model of the Distribution of Electron Density}

The model of electron density versus altitude used for the numerical calculations is illustrated in figure 4. Electron density $\left(N_{e} / \mathrm{cm}^{3}\right)$ is given on the right scale, and the corresponding local plasma frequency $\mathrm{f}_{p}$ in kilocycles can be read on the left scale.

In the exosphere, far above the earth's surface (above $1,000 \mathrm{~km}$ ), rather than $r^{-3}$, the distribution chosen was:

$$
N e / H=\text { constant }, \quad \text { or } \quad f_{p}^{2}=K f_{H},
$$

where $H$ is the local magnetic field strength and $f_{H}$ is the local electron gyrofrequency. Below 1,000 km, as a means of joining smoothly with a plausible ionospheric distribution, the above expression for $f_{p}^{2}$ is multiplied by the factor $\exp \left\{-(h-1,000)^{2} /\right.$ $\left.\mathscr{H}_{\text {ex }}^{2}\right\}$, where $h$ is the height in kilometers above the earth's surface. The scale height $\mathscr{H}_{\mathrm{ex}}$ is taken as $200 \mathrm{~km}$.

The ionospheric distribution is taken to be a Chapman-like layer

or

$$
N_{e}=N_{\max } \exp \left\{1 / 2\left(1-z-e^{-z}\right),\right.
$$

$$
f_{p}^{2}, \text { iono }=f_{p}^{2}, \max \exp \left\{1 / 2\left(1-z-e^{-z}\right)\right\},
$$

where $z=\left(h-h_{\max }\right) / \mathscr{H}$. The chosen value of $N_{\max }\left(10^{6}\right.$ $\mathrm{cm}^{-3}$ ) corresponds closely to the critical frequency of the F2 layer at the place and time of the observation to be matched (Boulder, Colorado, October 26, 1956, 2230 U'T or 1530 local standard time). The value of $h_{\max }$ was taken as $350 \mathrm{~km}$. Above $h_{\max }$ the

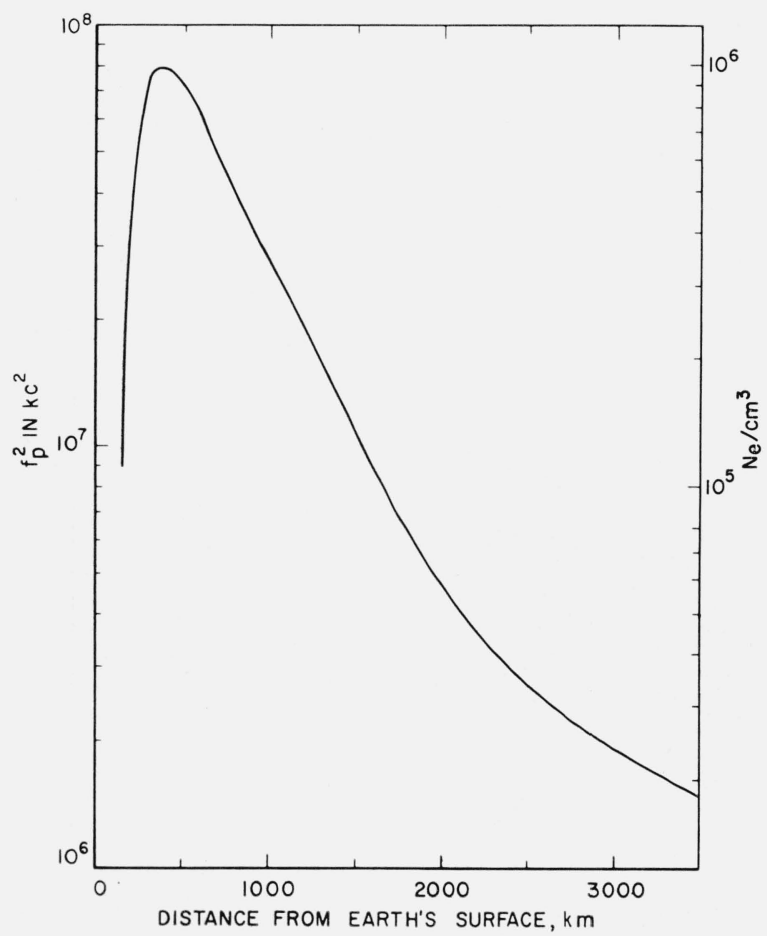

Figure 4. Distribution of electron density used for the theoretical calculation shown in figure 3. value of the scale height used was $\mathscr{H}_{A}=200 \mathrm{~km}$. Below $h_{\max }, \mathscr{H}_{B}=100 \mathrm{~km}$ was used.

Thus the total electron density for the model used is

$$
\begin{gathered}
f_{p}^{2}=f_{p, \text { Iono }}^{2}+f_{p, \text { exo }}^{2} \\
=f_{p, \text { max }}^{2} \exp \left\{1 / 2\left(1-z-e^{-z}\right)\right\} \\
+\left\{\begin{array}{ll}
K f_{H} \exp \left\{-(h-1,000)^{2} / \mathscr{H}_{e x}^{2}\right\} & \text { for } h<1,000 \mathrm{~km} \\
K f_{H} & \text { for } h>1,000 \mathrm{~km}
\end{array}\right\}
\end{gathered}
$$

Note that only a small range of values of the constant $K$ is possible if the electronic density in the exosphere is not to be too large near the earth (smaller than the lowest values of $N_{\max }$ ) nor too small to permit the propagation of whistlers at the greater distances. The value chosen here was 4,000 kc.

\subsection{Geometric Conditions}

The calculations were made for an ionized cloud of particles moving along the line of force which intersects the earth's surface at the geomagnetic latitude $\lambda=55^{\circ}$. The observations were made at Boulder at geomagnetic latitude $49^{\circ}$, but it is known from studies of whistlers that a signal propagating in the whistler mode can be received at places far from the foot of the nominal line of force along which the wave is propagated. For a dipole field, the line corresponding to $\lambda=55^{\circ}$ intersects the plane of the geomagnetic equator at 2.04 earth radii, or $13,010 \mathrm{~km}$ above the earth's surface. (Note that fig. 4 shows the model only out to the height $3,500 \mathrm{~km}$.) The total length along the line of force from the equator to the earth's surface is 3.17 earth radii, or 20,214 $\mathrm{km}$. At the equator the magnetic field strength along the line of force is a minimum. Its value is 0.011 gauss compared to 0.315 gauss at the surface. The corresponding gyrofrequency is $31.4 \mathrm{kc}$, and with the exosphere model (7) $N_{e}=1,560$ and $f_{p}=354$ kc at this height.

Using as the origin of time a starting position in the equatorial plane, the calculations were made for points regularly spaced along the trajectory to obtain: (1) the frequencies emitted; (2) the time of the emission; (3) the time for propagation of the wave from this point to the earth's surface along the remaining arc, using the full formula for group velocity in the whistler mode; and, (4) the sum of these two times to obtain the arrival time at the earth.

At $10,000 \mathrm{~km} / \mathrm{sec}$ the total time of flight for the cloud is nearly 2 sec while the range of arrival times of the emitted waves is about $1 / 2$ sec. Of course, one observes only the range of arrival times.

The "knee" in the "hook" in the present example corresponds to the transition at about $2,500-\mathrm{km}$ height between the electron density characteristic of the exosphere and the more rapidly increasing density in the ionosphere, which is responsible for the high branch of the "hook." 


\subsection{Velocity of the Cloud}

Generally speaking, the shape of the computed spectrum will remain approximately the same for different velocities. But the frequency and time scales will vary rapidly. A small velocity furnishes a range of arrival times that are too long and emitted frequencies that are too low. The durations and frequencies are very sensitive to the velocity. Velocities of about $1,000 \mathrm{~km} / \mathrm{sec}$, as suggested by the delay between related solar and terrestrial events, give frequencies roughly ten times too small and durations 10 times too long. Satisfactory fits are obtained only for velocities of the order of 10,000 $\mathrm{km} / \mathrm{sec}$. Such a high velocity seems to indicate that some process in the interaction between the earth's exosphere and the solar corpuscular radiations gives high local velocities to the small clouds responsible for the vlf emissions. These clouds are tentatively associated with the flashes of luminosity in active auroras.

It is significant that this velocity is of the same order as that required for protons to penetrate to the $100-\mathrm{km}$ level in the aurora.

TABLE 2. Summary of the conditions assumed for the sample theoretical calculation

\begin{tabular}{|c|c|c|}
\hline \multicolumn{2}{|c|}{ Distribution of electron density } & \multirow{2}{*}{ Other conditions } \\
\cline { 1 - 2 } Ionosphere & Exosphere & \\
\cline { 1 - 2 }$N_{\max }=10^{6} / \mathrm{cm}^{3}$ & $h_{\mathrm{ex}}=1,000 \mathrm{~km}$ & $\lambda=55^{\circ}$ \\
$h_{\mathrm{m} \mathrm{ax}}=350 \mathrm{~km}$ & $\mathscr{H}_{\mathrm{ex}}=200 \mathrm{~km}$ & $v_{s}=10^{4} \mathrm{~km} / \mathrm{s}$ \\
$\mathscr{H}_{A}=200 \mathrm{~km}$ & $K=4,000 \mathrm{kc}$ & \\
$\mathscr{H}_{B}=100 \mathrm{~km}$ & & \\
\hline
\end{tabular}

The work reported in this paper was supported partially by the Air Force Office of Research under Contract AF18 (603)-126. The helpful suggestions of T. N. Gautier and L. R. O. Storey are gratefully acknowledged.

\section{References}

[1] L. R. O. Storey, Ph. D. dissertation, Cambridge University, England, and Phil. Trans. Roy Soc. [A]246, 113 (1953).

[2] R. A. Helliwell, J. H. Crary, J. H. Pope, and R. L. Smith, The nose whistler, a new high latitude phenomenon, J. Geophys. Research 61, 139 (1956).

[3] G. R. Ellis, J. Atmospheric and Terrest. Phys. 8, 338 (1956).

[4] K. Maeda and I. Kimura, A theoretical investigation on the propagation path of the whistling atmospheries, Rep. Ionosphere Research Japan 10, 105 (1956).

[5] J. H. Pope, Diurnal variation in the occurrence of Dawn Chorus, Nature 180, 433 (1957).

[6] G. McK. Allcock, A study of the audio-frequency radio phenomenon known as Dawn Chorus, Australian J. Phys. 10, 286 (1957).

[7] H. E. Dinger, Whistling atmospherics, Nav. Research Lab. Rep. 4825 (September 14, 1956).

[8] J. M. Watts, An observation of audio-frequency electromagnetic noise during a period of solar disturbance, J. Geophys. Research 62, 199 (1957).

[9] G. R. Ellis, Low frequency radio emission from aurorae, J. Atmospheric and Terrest. Phys. 10, 302 (1957).

[10] J. R. Pierce, Traveling-wave Tubes (D. Van Nostrand Co., New York, N.Y., 1950).

[11] A. B. Meinel, Evidence for the entry into the upper atmosphere of high-speed protrons during auroral activity, Science, 112, 590 (1950).

[12] L. Vegard, Terrestrial magnetism and electricity, J. A. Fleming, ed., p. 609 (McGraw-Hill, New York, N.Y., 1937).

[13] Stanford University, Low-frequency propagation studies, pt. 1, Whistlers and related phenomena, Final report, Contract AF 19 (604)-795 (June 15, 1953 to September 30, 1956).

[14] D. F. Martyn, The theory of magnetic storms and auroras, Nature, 167, 92 (1951).

Boulder, Colo. (Paper 63D1-4). 